ARTigo Especial

SPECIAL ArTicLe

\title{
Osteoartrite (Artrose): Tratamento ${ }^{(*)}$
}

\author{
Coimbra IB, Pastor EH, Greve JMD, Puccinelli MLC, Fuller R, Cavalcanti FS, Maciel FMB, Honda E
}

\section{DESCRIÇÃO DO MÉTODO DE COLETA DE EVIDÊNCIAS}

Foram utilizados os estudos disponíveis na literatura médica presentes nas seguintes bases de dados, acessíveis através da internet: OVID (EBM-Reviews, incluindo-se as bases de dados da Cochrane) e o Medline, de 1966 até o presente, através do Pubmed. Foram selecionados trabalhos de meta-análise e estudos duplo-cegos randomizados, quando presentes. Relatos ou série de casos foram utilizados quando publicados em jornais de reconhecida idoneidade. As opiniões dos especialistas presentes foram utilizadas em relação a terapias não disponíveis na literatura e que fossem consideradas pela unanimidade dos presentes como importante para o manejo dos pacientes com osteoartrite. Envio prévio da bibliografia principal aos participantes. Reunião para elaboração do documento. Colocação do rascunho na internet por dez dias para mudanças. Elaboração final do documento.

\section{GRAU DE RECOMENDAÇÃO E FORÇA DE EVIDÊNCIA}

A: Estudos experimentais e observacionais de melhor consistência.

B: Estudos experimentais e observacionais de menor consistência.

C: Relatos de casos (estudos não controlados).

D: Opinião desprovida de avaliação crítica, baseada em consensos, estudos fisiológicos ou modelos animais.

\section{OBJETIVOS}

Conciliar informações e condutas referentes ao tratamento da osteoartrite pelas três principais especialidades envolvidas, reumatologia, fisiatria e ortopedia. As condutas consensuais para a maioria dos participantes foram então agrupadas e constam nas recomendações deste documento.

\section{CONFLITO DE INTERESSE}

Os autores Coimbra IB, Pucinelli MLC, Cavalcanti FS e Maciel $\mathrm{FMB}$, declararam vínculo com a Indústria Farmacêutica.

\section{INTRODUÇÃO}

A osteoartrite, doença articular degenerativa, artrose ou osteoartrose, como ainda é conhecida no nosso meio, é a doença reumática mais prevalente entre indivíduos com mais de 65 anos de idade. Estudos americanos apontam que mais de 50 milhões de pessoas apresentam hoje esta enfermidade. No Brasil, não existem dados precisos sobre esta prevalência. Os conhecimentos adquiridos recentemente no conhecimento da fisiopatogenia levaram a uma alteração no conceito desta doença. Antes se acreditava tratar-se de uma doença progressiva, de evolução arrastada, sem perspectivas de tratamento, encarada por muitos como natural do processo de envelhecimento. Hoje, no entanto, é vista como uma enfermidade em que é possível modificar o seu curso evolutivo, tanto em relação ao tratamento sintomático imediato, quanto ao seu prognóstico. É uma das causas mais freqüentes de dor do sistema músculoesquelético e de incapacidade para o trabalho no Brasil e no mundo. É uma afecção dolorosa das articulações que ocorre por insuficiência da cartilagem, ocasionada por um desequilíbrio entre a formação e a destruição dos seus principais elementos, associada a uma variedade de condições como: sobrecarga mecânica, alterações bioquímicas da cartilagem e membrana sinovial e fatores genéticos. A denominação mais aceita internacionalmente da doença é osteoartrite. O termo artrose ainda é muito utilizado, conhecido e associado aos aspectos mecânicos. É uma doença crônica,

\footnotetext{
* Trabalho realizado por representantes da Sociedade Brasileira de Reumatologia. Elaboração final: 29 de setembro de 2003.

O Projeto Diretrizes, iniciativa conjunta da Associação Médica Brasileira e Conselho Federal de Medicina, tem por objetivo conciliar informações da área médica a fim de padronizar condutas que auxiliem o raciocínio e a tomada de decisão do médico. As informações contidas neste projeto devem ser submetidas à avaliação e à crítica do médico, responsável pela conduta a ser seguida, frente à realidade e ao estado clínico de cada paciente a ser seguida, frente à realidade e ao estado clínico de cada paciente.
} 
multifatorial, que leva a uma incapacidade funcional progressiva. $O$ tratamento deve ser também multidisciplinar, e buscar a melhora funcional, mecânica e clínica e, por esta razão a realização deste consenso tornou-se necessária. Profissionais que lidam com a enfermidade, das áreas de reumatologia, fisiatria e ortopedia, reuniram-se para a sua elaboração.

\section{TRATAMENTO NÃO FARMACOLÓGICO}

\section{PROGRAMAS EDUCATIVOS ${ }^{(1)}$ (D)}

- Esclarecimento sobre a doença: salientar que a doença não é sinônimo de envelhecimento e está relacionada com a capacidade funcional, sendo que a intervenção terapêutica trará considerável melhora de qualidade de vida.

- Motivar e envolver o paciente no seu tratamento, pois o paciente é um agente ativo no seu programa de reabilitação.

- A prática de atividades esportivas deve ser estimulada, porém, sob orientação de um profissional habilitado.

- Orientação para cuidados com relação ao uso de rampas e escadas.

- Orientação com relação à ergonomia do trabalho doméstico e/ou profissional.

\section{EXERCÍCIOS TERAPÊUTICOS COM ORIENTAÇÃO (PRESCRIÇÃO INDIVIDUALIZADA PELA FISIOTERAPIA)}

- Fortalecimento - ganho de massa muscular. O fortalecimento do músculo quadríceps deve ser feito nas artrites do joelho ${ }^{(2)}(\mathbf{A})$.

- Aeróbios - Condicionamento físico ${ }^{(2)}(\mathbf{A})$.

- Alongamento - Flexibilidade, como parte da cinesioterapia $^{(3)}(\mathbf{A})$.

Órteses e equipamentos de auxílio à marcha também podem ser indicados quando há necessidade de melhorar, auxiliar ou substituir uma função ${ }^{(4)}(D)$.

Estabilização medial da patela, através de goteiras elásticas, é efetiva no tratamento da sintomatologia dolorosa da osteoartrite fềmuro-patelar ${ }^{(5)}(\mathbf{A})$.

Palmilhas anti-varo, associadas à estabilização de tornozelo, são eficientes na melhora da dor e função na osteoartrite do compartimento medial do joelho ${ }^{(6)}(\mathbf{A})$.

Agentes físicos - termoterapia ${ }^{(7)}(\mathbf{D})$, eletroterapia analgésica $^{(8)}(\mathbf{A})$ e o TENS ${ }^{(9)}(\mathbf{D})$ são meios coadjuvantes efetivos no tratamento sintomático da dor, embora as revisões sistemáticas apontem a necessidade de novos estudos com metodologia adequada. Em um estudo de revisão sistemática, o resultado com o uso do ultra-som não foi melhor que placebo $^{(10)}(\mathbf{A})$.

\section{TRATAMENTO FARMACOLÓGICO}

O tratamento sempre deve ter uma abordagem multifatorial, pois cada vez é mais claro que a prescrição medicamentosa isolada não é suficiente para o controle ideal da doença.

\section{ANALGÉSICOS E ANTIINFLAMATÓRIOS}

- Paracetamol em doses efetivas, isto é, até $4 \mathrm{~g} /$ dia, para se obter analgesia, principalmente em pacientes com manifestação leve ou moderada, é indicado como medicação de primeira escolha, ressaltando-se contudo não utilizá-lo em pacientes com história de hepatopatias ${ }^{(11)}(\mathbf{D})$. Segundo a experiência dos autores, a dipirona, em nosso meio disponível gratuitamente na rede básica de saúde, pode ser usada com o mesmo objetivo (D).

- Inibidores específicos da COX-2 $2^{(12)}($ D) ou os antiinflamatórios não seletivos, esses últimos associados a inibidor de bomba de prótons ou famotidina ${ }^{(13)}(\mathbf{A})$, podem ser indicados nos casos que apresentam quadro inflamatório evidente.

Em casos cujos fatores de risco estão presentes na tabela 1, devem ser utilizados os inibidores específicos da COX-2(A).

Opióides naturais ou sintéticos. Nos casos de má resposta terapêutica aos medicamentos anteriores, ou ainda, quando houver contra-indicação ao uso de inibidores específicos da COX-2 ou aos antiinflamatórios não seletivos, pode-se associar os opióides naturais ou sintéticos. Também em casos de pacientes em uso de antiinflamatórios e que apresentem reagudização da dor, os opióides como o tramadol podem ser utilizados ${ }^{(15)}(\mathbf{A})$

TABELA 1

FATORES DE RISCO ASSOCIADOS AO USO DE ANTIINFLAMATÓRIOS PARA EFEITOS ADVERSOS EM TRATO GASTROINTESTINAL ALTO ${ }^{(14)}(\mathbf{A})$

- Idade maior ou igual a 65 anos

- Outras condições médicas

- Glicocorticóides orais

- História de úlcera péptica

- História de sangramento gastrointestinal

- Anticoagulantes 


\section{AGENTES TÓPICOS}

- Capsaicina é um bom agente terapêutico para sintomatologia dolorosa ${ }^{(16)}(\mathbf{A})$, porém, os efeitos colaterais decorrentes do uso tópico, como a irritabilidade ocular ou epidérmica, limitam seu uso.

- Antiinflamatório não-hormonal tópico, como cetoprofeno, ibuprofeno, felbinaco e piroxicam, tem um efeito significativo no tratamento sintomático da dor aguda ou crônica $^{(17)}(\mathbf{A})$.

\section{DROGAS SINTOMÁTICAS DE AÇÃO DURADOURA}

São consideradas drogas de ação duradoura aquelas que têm ação prolongada na melhora da dor e cujo efeito terapêutico persiste mesmo após a sua suspensão. Estas drogas vêm se firmando na literatura como boas no tratamento sintomático da osteoartrite. As drogas disponíveis no mercado brasileiro são: sulfato de glucosamina, diacereína e extratos não saponificáveis de soja e abacate.

- Sulfato de glucosamina para o tratamento sintomático da osteoartrite de joelhos é usado na dose de $1,5 \mathrm{~g} / \mathrm{dia}^{(18)}(\mathbf{A})$.

- A cloroquina vem sendo utilizada em vários serviços brasileiros, com base na experiência pessoal dos especialistas, mostrando bons resultados. A indicação inicial foi para osteoartrite erosiva de mão e, posteriormente, passou a ser usada em outras formas da doença. Por tratar-se de droga com efeitos colaterais e que requer acompanhamento profilático para evitá-los, deverá apenas ser manuseada por profissionais treinados.

\section{TERAPIA INTRA-ARTICULAR}

- A infiltração intra-articular com triancinolona hexacetonida também pode apresentar controle da dor e da inflamação em casos com quadro inflamatório evidente ${ }^{(19)}(\mathbf{A})$.

- Uso intra-articular do ácido hialurônico está indicado para o tratamento da osteoartrite do joelho grau II e III nas fases aguda e crônica ${ }^{(20)}(\mathbf{D})$.

\section{REFERÊNCIAS}

1. Superio-Cabuslay E, Ward MM, Lorig KR. Patient education interventions in osteoarthritis and rheumatoid arthritis: a metaanalytic comparision with nonsteroidal antiinflammatory drug treatment. Arthritis Care Res 9:292-301, 1996.

2. Ettinger WH Jr, Burns R, Messier SP, et al. A randomized trial comparing aerobic exercise and resistance exercise with a health education program in older adults with knee osteoarthritis. The Fitness Arthritis and Seniors Trial (FAST). JAMA 277:25-31, 1997.

3. Van Baar ME, Dekker J, Oostendorp RA, et al. The effectiveness of exercise therapy in patients with osteoarthritis of the hip or knel: a randomized clinical trial. J Rheumatol 25:2432-9, 1998.

\section{TRATAMENTO CIRÚRGICO}

Os pacientes com osteoartrite grau II e III com comprometimento progressivo da independência das atividades de vida diária e falha do tratamento conservador devem ser referidos para o ortopedista que fará a indicação do tratamento cirúrgico. As cirurgias indicadas são: desbridamento artroscópico, osteotomias e artroplastias.

\section{OSTEOTOMIAS}

É importante se ressaltar o papel das osteotomias, pois são procedimentos que devem ser feitos precocemente em pacientes selecionados ${ }^{(21)}(\mathbf{A})$. São feitos dois tipos de osteotomias:

- Profilática - indicada precocemente em pacientes sintomáticos e ainda sem alterações radiográficas para a correção dos desvios de eixos articulares.

- Terapêutica - indicada em casos sintomáticos e com alterações radiográficas. É feita para modificar o eixo de alinhamento do membro afetado e deslocar a carga para outra região da superfície articular.

\section{DESBRIDAMENTO ARTROSCÓPICO}

$\mathrm{Na}$ experiência dos autores, são indicações precisas para correção das lesões parciais de meniscos, labrum e retirada de corpos livres intra-articulares da mesma forma que para as osteoartroses de quadril ${ }^{(22)}(\mathbf{C})$.

\section{ARTROPLASTIAS}

$\mathrm{Na}$ experiência dos autores, as artroplastias totais promovem acentuada redução na dor e melhora funcional na maioria de casos selecionados da doença.

\section{ARTRODESES}

Indicada principalmente na dor e incapacidade funcional persistentes da osteoartrite de tornozelos e que não tenha melhorado com tratamento conservador ${ }^{(23)}(\mathbf{D})$.

4. Pendleton A, Arden N, Dougados M, et al. EULAR recommendations for the management of knee osteoarthritis: report of a task force of the Standing Committee for International Clinical Studies Including Therapeutic Trials (ESCISIT). Ann Rheum Dis 59:936-44, 2000.

5. Cushnaghan J, McCarthy C, Dieppe P. Taping the patella medially: a new treatment for osteoarthritis of the knee joint? BMJ 308: 753-5, 1994.

6. Toda Y, Segal N, Kato A, Yamamoto S, Irie M. Effect of a novel insole on the subtalar joint of patients with medial compartment osteoarthritis of the knee. J Rheumatol 28:2705-10, 2001.

7. Rush PJ, Shore A. Physician perceptions of the value of physical modalities in the treatment of musculoskeletal disease. $\mathrm{Br} J \mathrm{R}$ heumatol 33:566-8, 1994. 
8. Hulme J, Robinson V, DeBie R, Wells G, Judd M, Tugwell P. Electromagnetic fields for the treatment of osteoarthritis. Cochrane Database Syst Rev1: CD003523, 2002.

9. Osiri M, Welch V, Brosseau L, et al. Transcutaneous electrical nerve stimulation for knee ostearthritis. Cochrane Database Syst Rev 4:CD002823, 2000.

10. Welch V, Brosseau L, Peterson J, Shea B, Tugwell P, Wells G. Therapeutic ultrasound for osteoarthritis of the knee. Cochrane Database Syst Rev 3:CD003132, 2001.

11. Eccles M, Freemantle N, Mason J. North of England evidence based guideline development project: summary guideline for non-steroidal anti-inflammatory drugs versus basic analgesia in treating the pain of degenerative arthritis. The North of England non-steroidal antiinflammatory Drug Guideline Development Group. BMJ 317: 526-30, 1998.

12. Hawkey CJ. COX-2 inhibitors. Lancet 353:307-14, 1999.

13. Taha AS, Hudson N, Hawkey CJ, et al. Famotidine for the prevention of gastric and duodenal ulcers caused by non-steroidal antiinflammatory drugs. N Engl J Med 334:1435-9, 1996.

14. Gabriel SE, Jaakkimainen L, Bombardier C. Risk for serious gastrointestinal complications related to use of nonsteroidal antiinflammatory drugs: a meta-analysis. Ann Intern Med 115:787-96, 1991.

15. Roth SH. Efficacy and safety of tramadol $\mathrm{HCl}$ in breakthrough musculoskeletal pain attributed to osteoarthritis. J Rheumatol 25:1358-63, 1998.
16. Zhang WY, Li Wan Po A. The effectiveness of topically applied capsaicin: a meta-analysis. Eur J Clin Pharmacol 46:517-22, 1994.

17. Moore RA, Tramer MR, Carroll D, Wiffen PJ, McQuay HJ. Quantitative systematic review of topically applied nonsteroidal antiinflammatory drugs. BMJ 316:333-8, 1998.

18. Reginster JY, Deroisy R, Rovati LC, et al. Long-term effects of glucosamine sulphate on osteoarthritis progression: a randomized, placebo-controlled clinical trial. Lancet 357:251-6, 2001.

19. Gaffney K, Ledingham J, Perry JD. Intra-articular triamcinolone hexacetonide in knee osteoarthritis: factors influencing the clinical response. Ann Rheum Dis 54:379-81, 1995.

20. Raynauld JP, Torrance GW, Band PA, et al. A prospective, randomized, pragmatic, health outcomes trial evaluating the incorporation of hylan G-F 20 into the treatment paradigm for patients with knee osteoarthritis (Part 1 of 2): clinical results. Osteoarthritis Cartilage 10:506-17, 2002.

21. Schramm M, Pitto RP, Rohm E, Hohmann D. Long-term results of spherical acetabular osteotomy. J Bone Joint Surg Br 81:60-6, 1999.

22. Margheritini F, Villar RN. The efficacy of arthroscopy in the treatment of hip osteoarthritis. Chir Organi Mov 84:257-61, 1999.

23. Abidi NA, Gruen GS, Conti SF. Ankle arthrodesis: indications and techniques. J Am Acad Orthop Surg 8:200-9, 2000. 